\title{
Prediction Models of Retention Indices for Increased Confidence in Structural Elucidation during Complex Matrix Analysis: Application to Gas Chromatography Coupled with High Resolution Mass Spectrometry
}

Eric Dossin, Elyette Martin, Pierrick Diana, Antonio Castellon, Aurelien Monge, Pavel Pospisil, Mark Bentley, Philippe A. Guy*

Philip Morris International R\&D, Philip Morris Products S.A., Quai Jeanrenaud 5, CH-2000 Neuchatel, Switzerland. (part of Philip Morris International group of companies)

\section{$\underline{\text { Table of contents }}$}

\section{Supporting information Figures (Word document)}

Supplementary figure 1. Plot of chemical space of compounds reported in tobacco and tobacco smoke a) AlogP against molecular weight, b) boiling point against AlogP.

Supplementary figure 2. Data comparison of LRI values across different laboratories plotted against our experimental results.

Final Compound Scoring: Formula used to calculate the final score for each compound putative hit reported in Agilent Technologies software (cf. Figure 4 of the main manuscript). This calculation combines NIST14 score with LRI experimental value against those predicted from RM and CG.

\section{Supporting information 1 (Excel document)}

Spreadsheet 1: Description of the reference standards used for the training set $(n=401)$ and results.

Spreadsheet 2: Description of the reference standards used for the test set $(n=151)$ and results.

Spreadsheet 3: Description of the additional identified compounds $(n=23)$ and results.

Spreadsheet 4: Calculation of LRI values.

Spreadsheet 5: Description of the reference standards used as benchmarking our experimental LIR against other laboratories.

\section{Supporting information 2 (Excel document)}

Spreadsheet 1: List of Dragon descriptors used to build RapidMiner model and results for the model optimization.

Spreadsheet 2: Results for the model optimization for ChromGenius software.

Spreadsheet 3: Classification of the reference standards used for the test and validation sets.

Supporting information for RapidMiner protocol (xml document)

Supporting information_RapidMiner protocol 
Supplementary figure 1: Plot of the chemical space of 8,665 compounds already reported in tobacco and tobacco smoke as the coefficient of partitioning between octanol and water (ALogP method

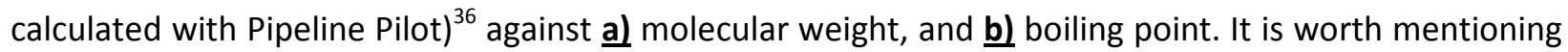
that 29 compounds have not been included as their masses ranged above $m / z 1,000$. Moreover, compounds having combined functionalities have been numbered several times.

a)

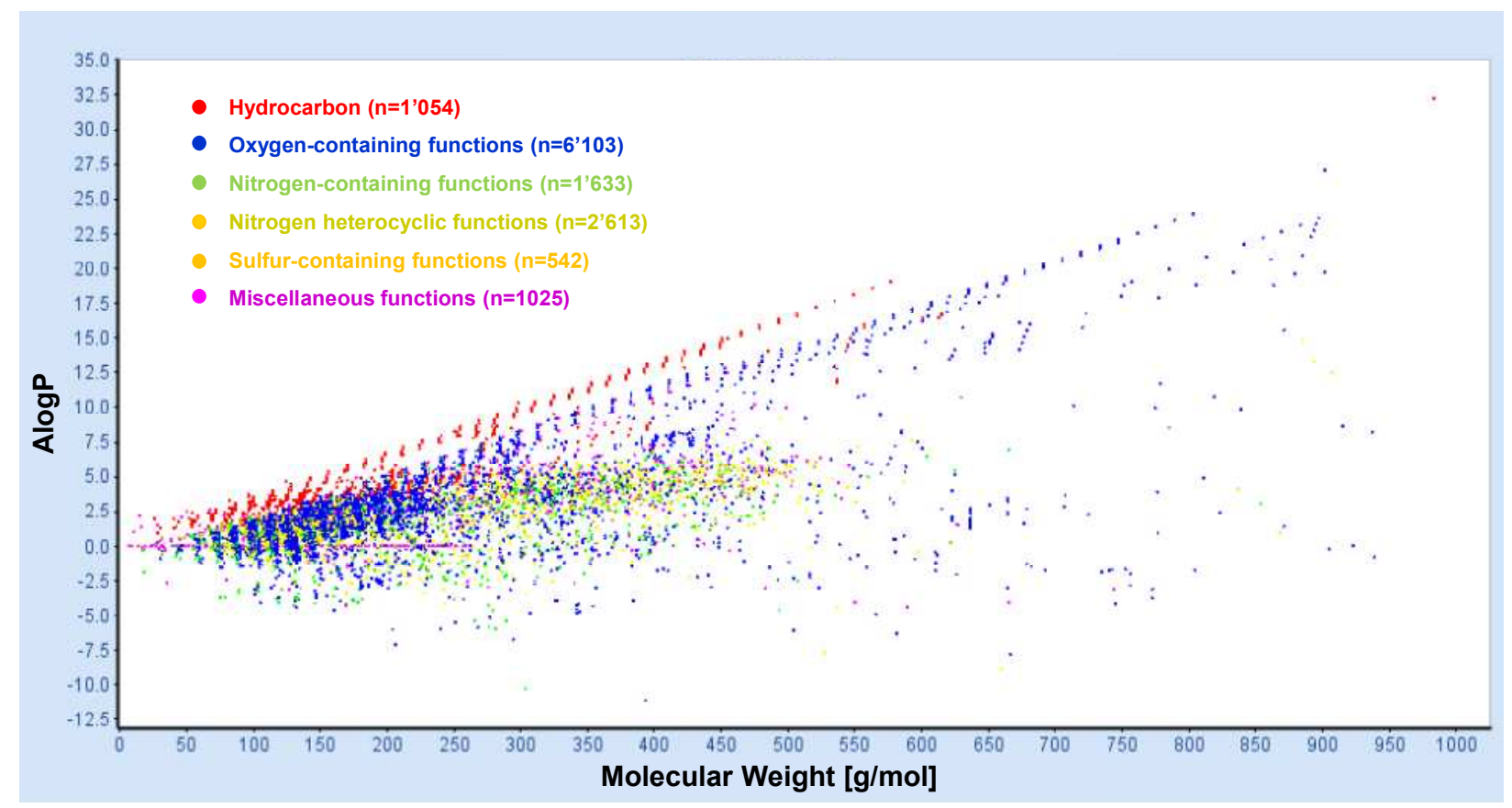

b)

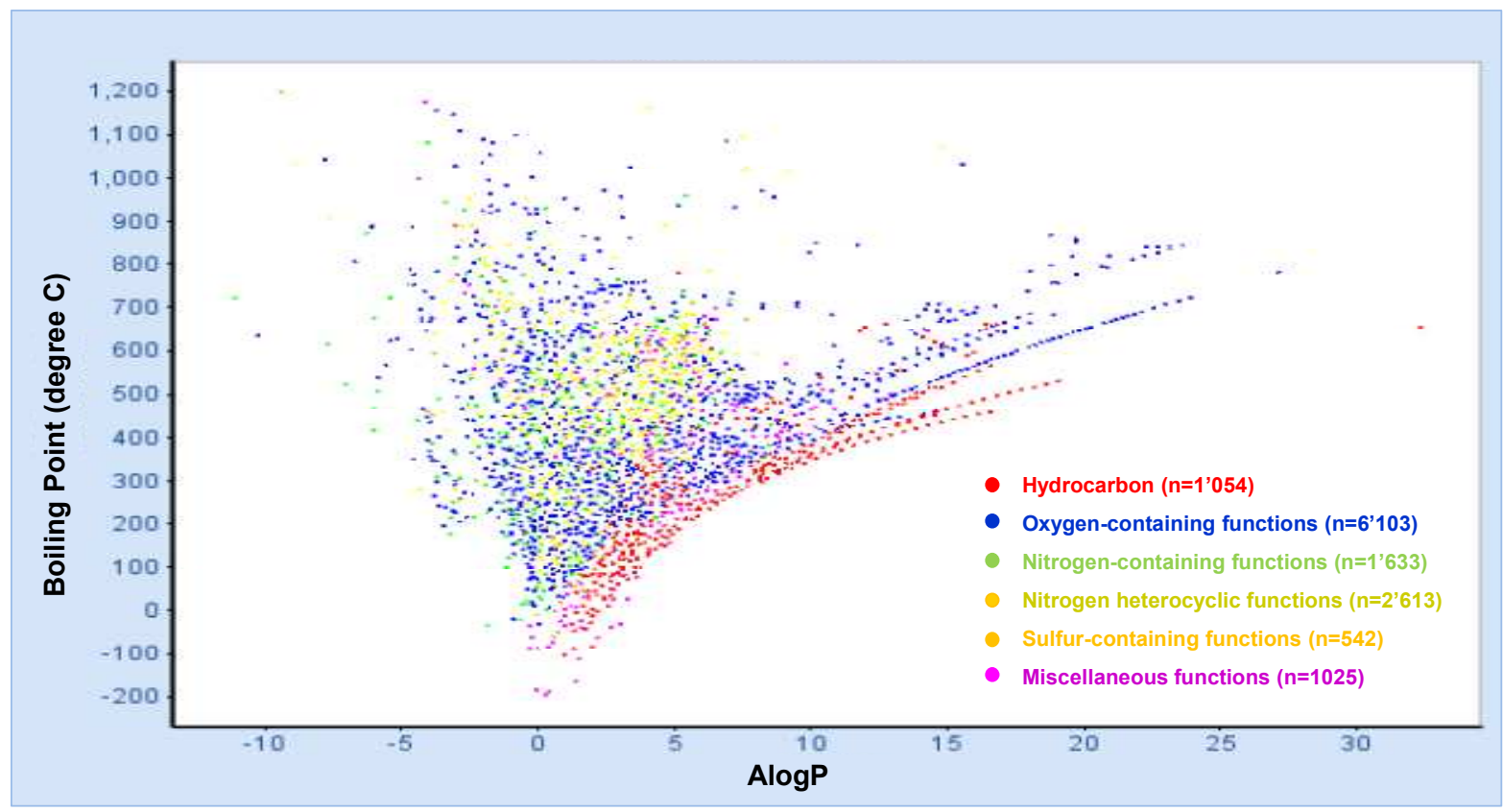


Supplementary figure 2: Data comparison of LRI values across different laboratories plotted against our experimental results. a) LRI data of three laboratories, ${ }^{38-40}$ have been respectively highlighted as lines, circles, and triangles. b) LRI differences between externally published minus our experimental results have been plotted against our experimental values in which the odd n-alkanes (retention index markers) have been highlighted. The full description of chemicals has been reported in supporting information_1 (spreadsheet identified as matching ext data).
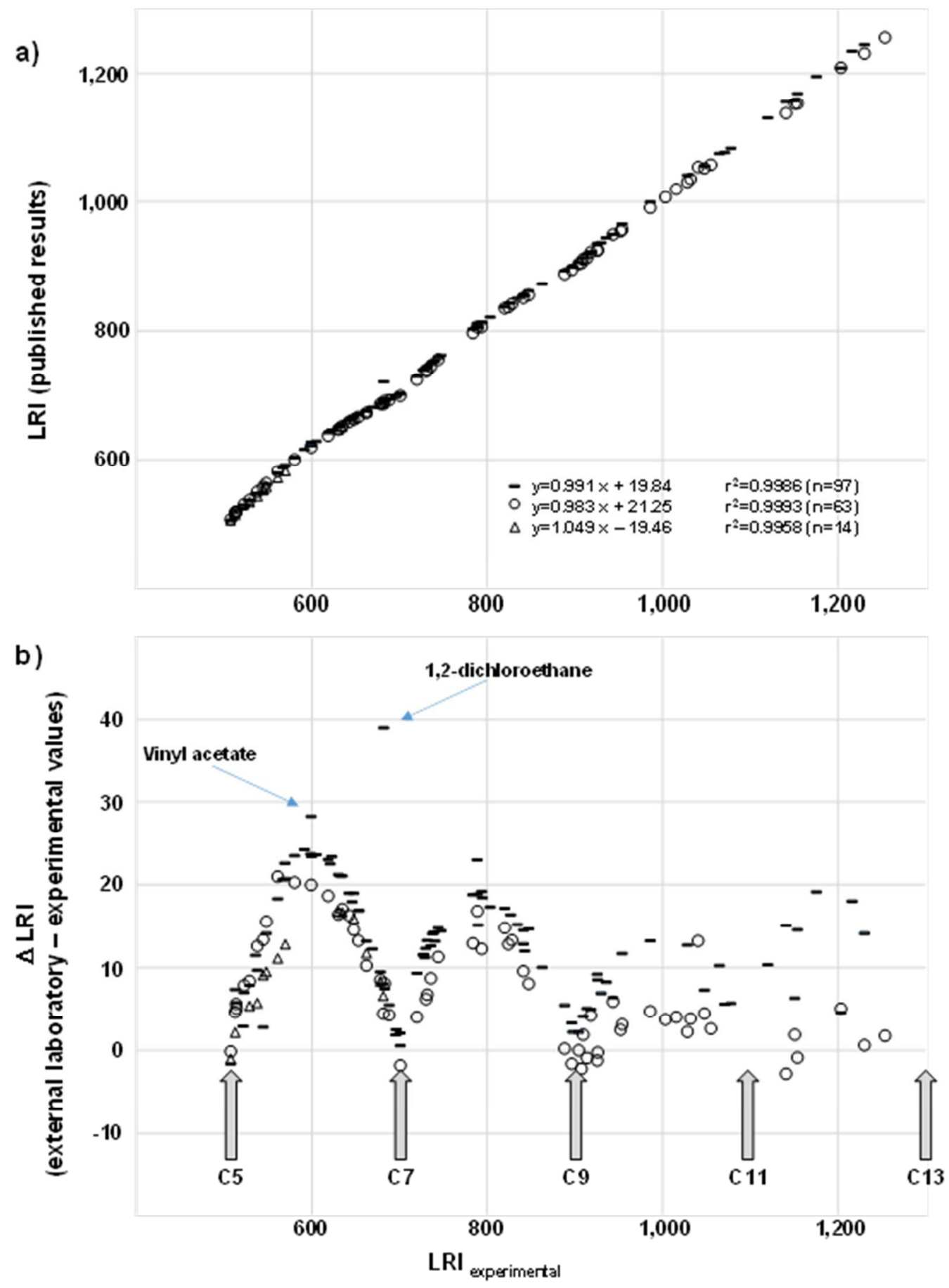
Final Compound Scoring: Formula used to calculate the final score for each compound putative hit reported in Agilent Technologies software (cf. Figure 4 of the main manuscript). This calculation combines NIST14 score with LRI experimental value against those predicted from RM and CG.

$$
\begin{aligned}
& \text { Final Score }=\frac{\text { NIST14 Score } x L_{R I_{\text {exp }}{ }^{2}}}{\underline{\left(L R I_{\text {exp }}+\sqrt[2]{L R I_{\text {pred }} \text { cG }}-L R I_{\text {exp }}\right)}} \\
& \frac{\left(L R I_{\text {exp }}+\sqrt[2]{L R I_{\text {pred } R M}-L R I_{\text {exp }}}\right)}{(L R I}
\end{aligned}
$$

